\title{
Chlamydia pneumoniae-associated pleuropericarditis: a case report and systematic review of the literature
}

\author{
Konstantinos G. Kyriakoulis ${ }^{*}{ }^{*}$, Anastasios Kollias ${ }^{\dagger}$, George E. Diakos, loannis P. Trontzas, Eleni Fyta, \\ Nikolaos K. Syrigos and Garyphallia Poulakou
}

\begin{abstract}
Background: Chlamydia pneumoniae is a common cause of atypical community acquired pneumonia (CAP). The diagnostic approach of chlamydial infections remains a challenge. Diagnosis of delayed chlamydial-associated complications, involving complex autoimmune pathophysiological mechanisms, is still more challenging. C. pneumoniaerelated cardiac complications have been rarely reported, including cases of endocarditis, myocarditis and pericarditis.

Case presentation: A 40-year old female was hospitalized for pleuropericarditis following lower respiratory tract infection. The patient had been hospitalized for CAP (fever, dyspnea, chest X-ray positive for consolidation on the left upper lobe) 5 weeks ago and had received ceftriaxone and moxifloxacin. Four weeks after her discharge, the patient presented with fever, shortness of breath and pleuritic chest pain and was readmitted because of pericardial and bilateral pleural effusions (mainly left). The patient did not improve on antibiotics and sequential introduction of colchicine and methylprednisolone was performed. The patient presented impressive clinical and laboratory response. Several laboratory and clinical assessments failed to demonstrate any etiological factor for serositis. Chlamydial IgM and IgG antibodies were positive and serial measurements showed increasing kinetics for lgG. Gold standard polymerase chain reaction of respiratory tract samples was not feasible but possibly would not have provided any additional information since CAP occurred 5 weeks ago. The patient was discharged under colchicine and tapered methylprednisolone course. During regular clinic visits, she remained in good clinical condition without pericardial and pleural effusions relapse.
\end{abstract}

Conclusions: C. pneumoniae should be considered as possible pathogen in case of pleuritis and/or pericarditis during or after a lower respiratory tract infection. In a systematic review of the literature only five cases of $C$. pneumoniae associated pericarditis were identified. Exact mechanisms of cardiovascular damage have not yet been defined, yet autoimmune pathways might be implicated.

Keywords: Pericarditis, Pleuritis, Pleuropericarditis, Serositis, Chlamydia pneumonia, Community acquired pneumonia, Antigenic mimicry

\footnotetext{
*Correspondence: konkyriakoulis@gmail.com

${ }^{\dagger}$ Konstantinos G. Kyriakoulis and Anastasios Kollias have contributed equally to this work and share first authorship.

Third Department of Medicine, School of Medicine, National and Kapodistrian University of Athens, Sotiria Hospital, 152 Mesogion Avenue, 11527 Athens, Greece
}

\begin{abstract}
Background
Chlamydia pneumoniae is a common cause of atypical lower respiratory tract infection [1]. Cardiac complications related to C. pneumoniae infection have been rarely reported. These reports include cases of endocarditis, myocarditis or pericarditis during or after C. pneumoniae pneumonia [2-11]. Atherosclerosis has also been
\end{abstract}

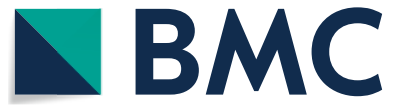

(c) The Author(s) 2021. Open Access This article is licensed under a Creative Commons Attribution 4.0 International License, which permits use, sharing, adaptation, distribution and reproduction in any medium or format, as long as you give appropriate credit to the original author(s) and the source, provide a link to the Creative Commons licence, and indicate if changes were made. The images or other third party material in this article are included in the article's Creative Commons licence, unless indicated otherwise in a credit line to the material. If material is not included in the article's Creative Commons licence and your intended use is not permitted by statutory regulation or exceeds the permitted use, you will need to obtain permission directly from the copyright holder. To view a copy of this licence, visit http://creativecommons.org/licenses/by/4.0/. The Creative Commons Public Domain Dedication waiver (http://creativeco mmons.org/publicdomain/zero/1.0/) applies to the data made available in this article, unless otherwise stated in a credit line to the data. 
related to C. pneumoniae infection [12-14]. We present the case of a 40-year old woman who suffered serositis (pleuropericarditis) after being treated for community acquired pneumonia (CAP). The diagnosis of C. pneumoniae serositis was established based on the kinetics of chlamydial antibodies over serial measurements. A systematic review of the literature was also performed in line with the PRISMA recommendations (www.prismastatement.org) to detect similar cases.

\section{Case presentation}

A 40-year old female was transferred to our Department of Medicine for the assessment and treatment of pleuropericarditis following lower respiratory tract infection. Her body mass index was $25.7 \mathrm{~kg} / \mathrm{m}^{2}$ and her personal history was positive for current smoking ( 10 cigarettes/day, 40 pack years) and arterial hypertension (occasional use of amlodipine $5 \mathrm{mg}$ ). The present history of the patient begins 5 weeks ago with a hospitalization in a Respiratory Department for CAP (fever, dyspnea, chest X-ray positive for consolidation on the left upper lobe; Additional File 1 ). The patient received ceftriaxone and moxifloxacin for 10 days without any medical report on causative pathogen. Four weeks after her discharge the patient presented with fever, shortness of breath especially in exertion and pleuritic chest pain and was admitted to a Respiratory Department where bilateral pleural (mainly left) and mild pericardial effusion were found. Pericardial friction rub was not identified. Her blood tests revealed increased neutrophils and indices of inflammation (Table 1, Additional File 1). Piperacillin/tazobactam and linezolid were initially administered. Thoracocentesis on the left side revealed neutrophilic exudate with $\mathrm{pH}$ 7.40. Despite antibiotic treatment, patient was not improved. Moreover C-reactive protein (CRP), pleural and pericardial effusions remained unchanged. Procalcitonin, high-sensitive troponin and brain natriuretic peptide levels were within normal limits during hospitalization. Blood and sputum cultures were negative. Extensive rheumatological workup was negative. In electrocardiography sinus tachycardia (112 bpm), frequent premature ventricular contractions, periods of ventricular trigeminy and inverted T-wave in V1-V6 were observed. Echocardiography was positive for mild mitral and triscupid valve regurgitation and mild pericardial effusion, without any sign of hemodynamic decompensation. Computed tomography (CT) imaging (including CT pulmonary angiogram) did not reveal pulmonary embolism or pathological findings from lung parenchyma and was positive only for the presence of pleural and pericardial effusions (Additional File 1). Pulmonologists administered ibuprofen $600 \mathrm{mg}$ three times daily with the indication of idiopathic pleuropericarditis. The patient presented allergic reaction to ibuprofen and was transferred to our Department of Medicine on day (D) 12. Upon her admission, the patient presented with diffuse maculo-papular rash that was attributed to the allergic reaction to ibuprofen. Ibuprofen was withdrawn and IV methylprednisolone $40 \mathrm{mg}$ once daily was initiated. Antibiotic treatment was suspended due to the lack of evidence of bacterial infection. Due to sinus tachycardia and premature ventricular complexes metoprolol $25 \mathrm{mg}$ twice daily was administered. Patient's file was reviewed and pericarditis investigation and differential diagnosis was conducted according to current guidelines [15]. The patient fulfilled two out of four major criteria (pericardial chest pain and new pericardial effusion), along with one of the additional supporting findings (elevated CRP, white blood cells and erythrocyte sedimentation rate), rendering the diagnosis of pericarditis evidence-based [15]. Colchicine $0.5 \mathrm{mg}$ once daily (patient's weight $66 \mathrm{~kg}$ ) was initiated, for the treatment of pericarditis under investigation. The patient presented clinical improvement and CRP dropped to nearly normal (Additional File 1). Methylprednisolone was stopped after 3 days, as it was administered to treat the allergic reaction to ibuprophen. Low grade fever and pleuritic chest pain recurred rapidly, along with rising CRP levels. A new chest X-ray revealed bilateral pleural effusion but this time mainly on the right side. A repeat thoracocentesis revealed neutrophilic exudate with $\mathrm{pH} 7.40$ and in a new chest $\mathrm{CT}$ the only new finding was the reduction of the left pleural effusion in combination with the increase of the right sided pleural effusion. Empirical initiation of meropenem was decided (Additional File 1). Clinical and laboratory findings did not improve with the addition of antibiotics; thus, antibiotics were suspended due to lack of evidence in favor of a bacterial cause for the new fever and IV methylprednisolone $40 \mathrm{mg}$ once daily was again initiated.

The patient presented impressive clinical and laboratory response to the combination of colchicine and methylprednisolone and was discharged (D 31) with the diagnosis of pleuropericarditis under investigation on colchicine $0.5 \mathrm{mg}$ once daily and methylprednisolone $16 \mathrm{mg}$ twice daily per os, while waiting for further laboratory test results. C. pneumoniae IgM and IgG antibodies [enzyme-linked immunosorbent assay (ELISA) VIRION/ SERION ELISA kit] were both positive upon admission of the patient to our Department on D 12 (IgM $20 \mathrm{U} /$ $\mathrm{ml}$, IgG $14 \mathrm{U} / \mathrm{ml}$ with normal limits $<15 \mathrm{U} / \mathrm{ml}$ for IgM and $<12 \mathrm{U} / \mathrm{ml}$ for IgG) (Fig. 1). Mycoplasma pneumoniae antibody titers were found to be negative, and a possible cross reaction, observed in $30 \%$ of patients with M. pneumoniae infection, was excluded [16]. In addition, a positive aspergillus antigen test (galactomannan) was found. Clinical and imaging findings were against aspergillosis, 


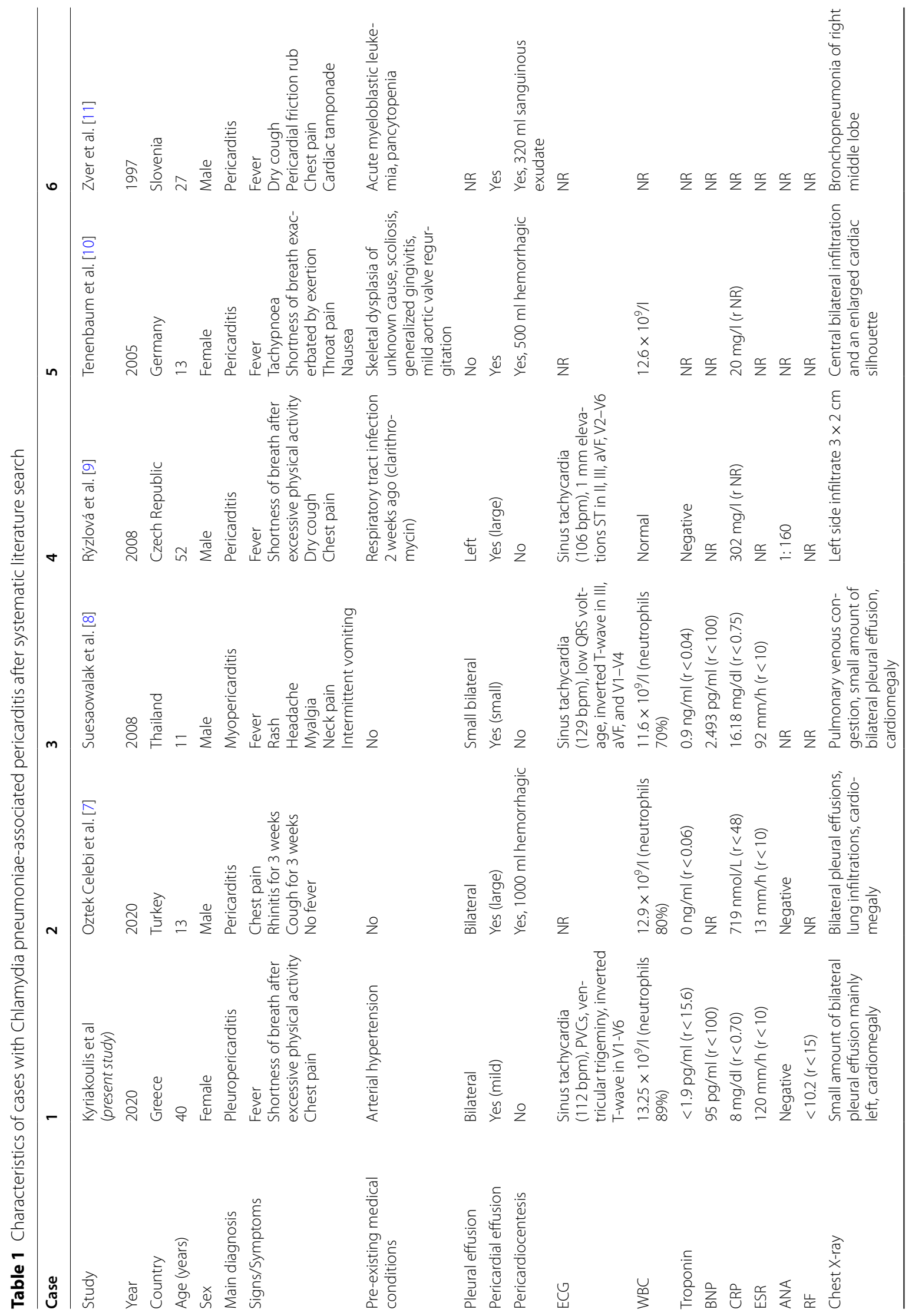




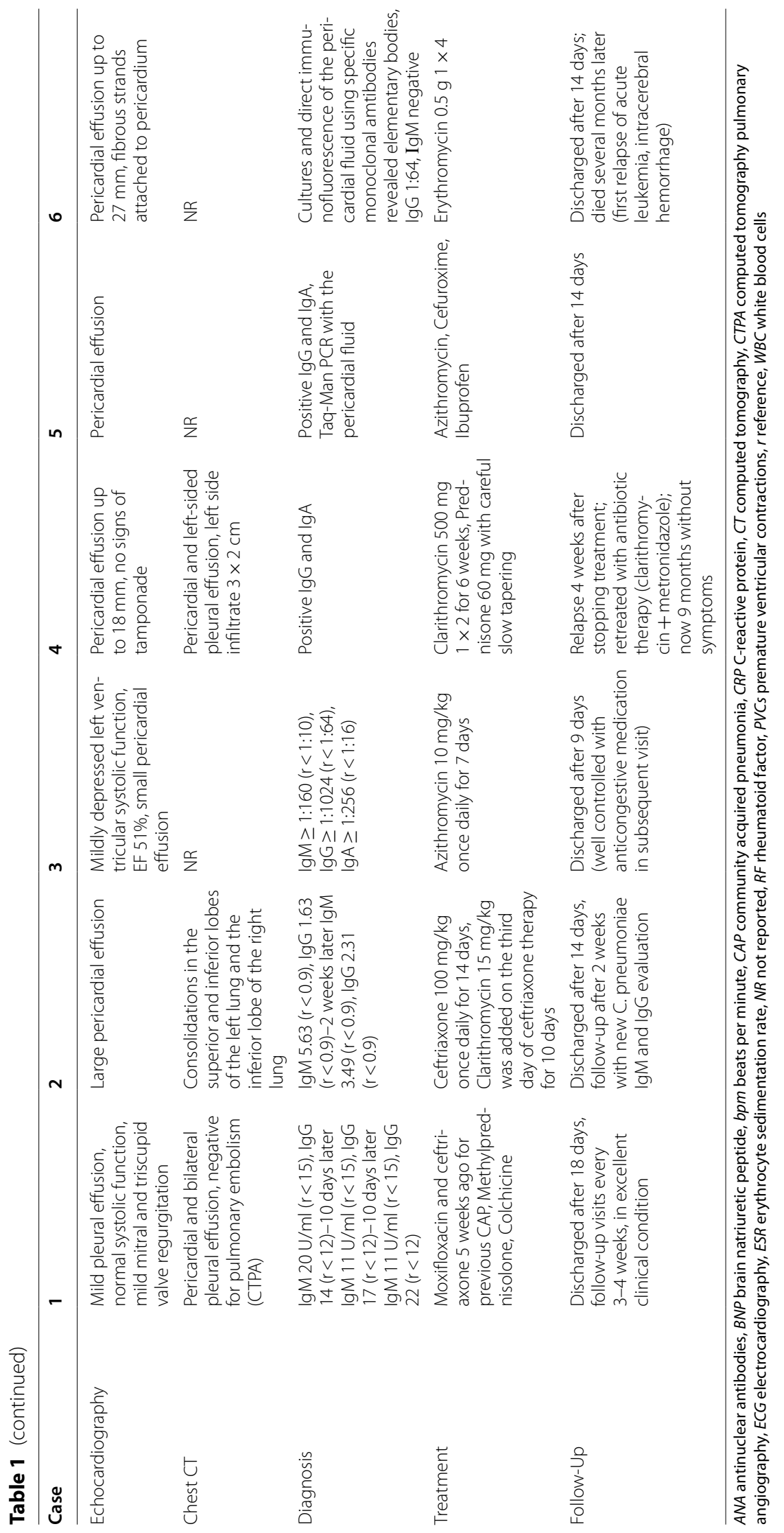




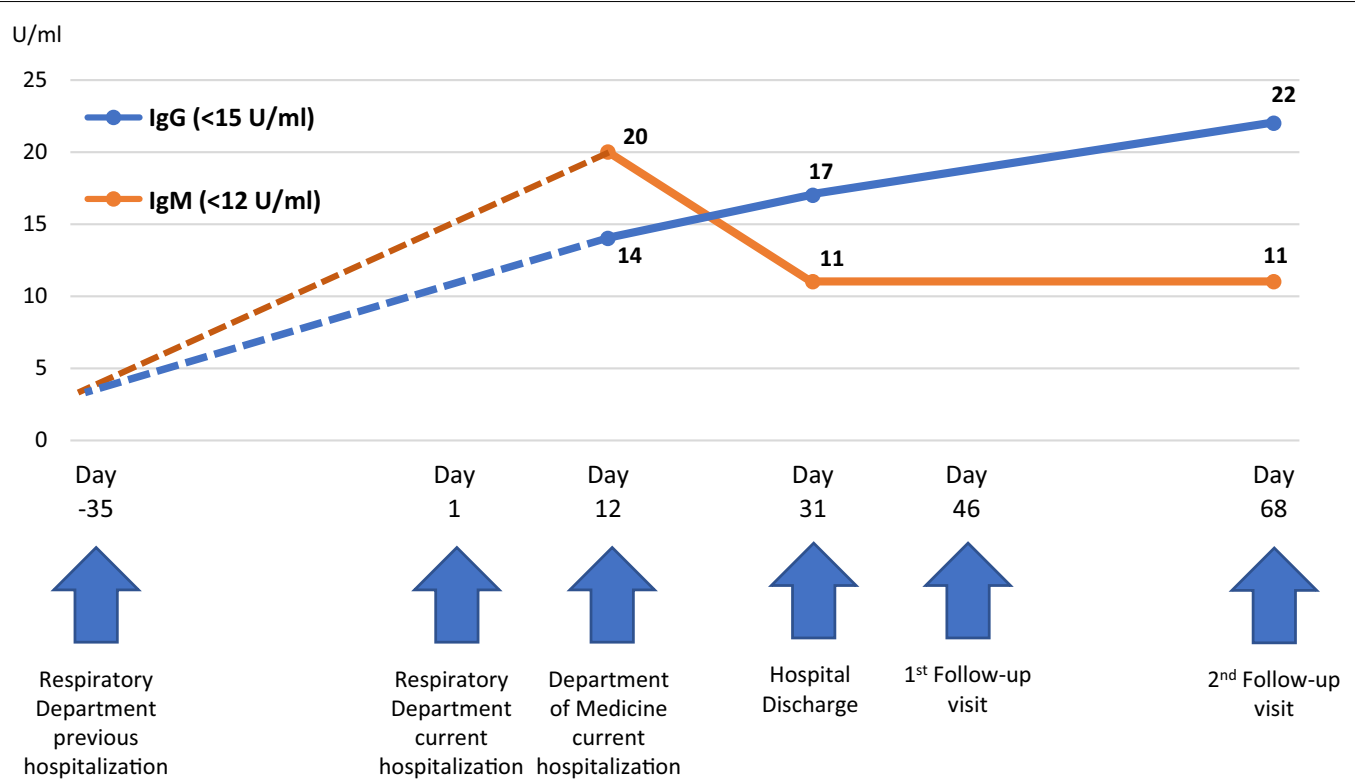

Fig. 1 Chlamydia pneumoniae lgM and lgG antibodies kinetics

the patient was not in an immunodeficient status, and this finding was considered as a false positive finding, probably due to prior treatment with piperacillin/tazobactam [17]. In order to follow the antibody kinetics, new antibody titers were ordered upon patient's discharge on D 31 (IgM $11 \mathrm{U} / \mathrm{ml}$, IgG $17 \mathrm{U} / \mathrm{ml}$ ) (Fig. 1). Thus, the combination of the patient's history and course, the negative extensive work up for other infections and autoimmune diseases, the negative imaging for pulmonary embolism and malignancies, along with the trend of the antibody kinetics rendered a diagnosis of C. pneumoniae related pleuropericarditis rather possible.

The patient visited the hospital on an outpatient basis two weeks after discharge (D 46). She was in good clinical condition, with normal laboratory values (white blood cells, CRP) and improved imaging evaluation (mild pleural effusions in CT imaging) (Additional File 1). Tapering of methylprednisolone was decided by reducing $8 \mathrm{mg}$ of methylprednisolone every 7 days. The patient presented to the next scheduled follow-up visit as outpatient one month later (D 68) on colchicine $0.5 \mathrm{mg}$ once daily and methylprednisolone $8 \mathrm{mg}$ once daily. She reported recurrence of mild pleuritic chest pain and low-grade fever. CRP was $2.8 \mathrm{mg} / \mathrm{dl}$ (normal limits $<0.7 \mathrm{mg} / \mathrm{dl}$ ) and imaging (echocardiography) revealed small pericardial effusion. Aggressive tapering of methylprednisolone was considered as the most probable cause of the clinical relapse of the patient. Uptitration of methylprednisolone to $16 \mathrm{mg}$ twice daily was decided. A third sample for $C$. pneumoniae antibodies was acquired (IgM $11 \mathrm{U} / \mathrm{ml}$, IgG $22 \mathrm{U} / \mathrm{ml}$ ) (Fig. 1). The patient was reassessed on D 105 in good clinical condition and absence of pleural effusion in chest X-ray and closer follow-up was planned for a slower tapering of corticosteroids. Complete rheumatologic evaluation was repeated (clinical and laboratory) without evidence of rheumatological disease.

Concerning the patient's perspective, she was informed of all the investigations performed and she was compliant with the interventions. She was relieved with the exclusion of severe causes (malignant) and fully aware of the need for regular follow-up. The patient engaged gradually to her daily activities without any concern.

\section{Discussion and conclusions}

The case of a 40-year old woman with pleuropericarditis associated with prior C. pneumoniae CAP has been described. Cardiac complications after or during Chlamydia infection (either C. pneumoniae, psittaci or trachomatis) have been described through the literature, though cases are rare, and mechanisms understudied. Most data are derived from non-human studies [12], while the association of Chlamydia infection with cardiac complications has been based mainly on cross-sectional studies with single antibody measurements, rather than multiple follow-up antibody titers and kinetics, like in our case $[18,19]$. Cardiac involvement seems to be even rarer when it comes to C. pneumoniae infection [2-11]. Previous reports associated with M. pneumoniae atypical CAP have also been reported and seem to be more common through the literature [20].

A systematic review of the literature was conducted independently by two investigators (GED, EF) to detect 
similar cases. Search algorithm and flowchart of included studies are shown in Fig. 2. Similar case reports are rare in the literature. Five studies have been identified [7-11]. Main characteristics of included studies are described in Table 1. The origin of the studies was Europe and Asia, while the prognosis of patients involved was good in most cases. The study by Oztek Celebi et al. [7] was the only one where more than one antibody measurements were ordered (two in total), in order to investigate a similar case involving a 13-year-old boy with pericardial and bilateral pleural effusions. However, antibody titers did not clearly follow the expected kinetics. Our study is unique in this context as more measurements of chlamydial antibodies were ordered (three in total) with typical fluctuations indicating past infection. Regarding treatment choices among the similar studies identified, most patients have been treated mainly with macrolides but there seems to be a lack of evidence regarding the use of colchicine and/or corticosteroids in their treatment (Table 1).

In our case the patient presented to our hospital with pleuropericarditis five weeks after hospitalization for CAP in another hospital, where she was treated with ceftriaxone and moxifloxacin without any report on causative pathogen. The kinetics of the IgM and IgG antibodies against $C$. pneumoniae indicated the latter as the causative microorganism (Fig. 1, Additional File 1). The patient was administered moxifloxacin in her first hospitalization. This is considered as an acceptable first-line empiric treatment for most patients with community-acquired pneumonia since the etiology is usually unknown at the time of diagnosis. The patient responded and was discharged. However, macrolides represent the first-line option for C. pneumoniae [21]. Whether an initial course

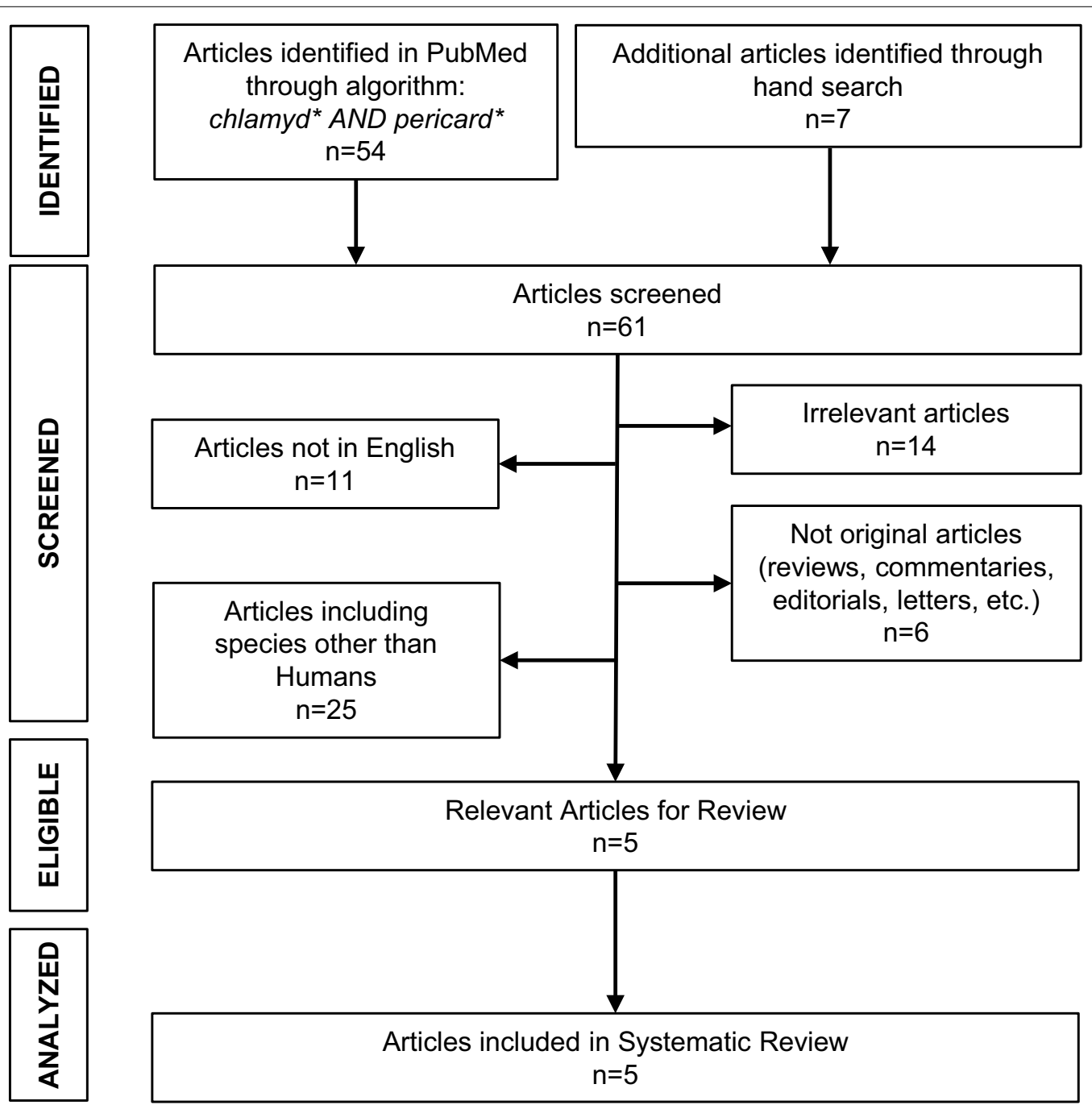

Fig. 2 Search algorithm and flowchart of studies included in systematic review 
with macrolides could avert the subsequent pericarditis is not known. So far, the documentation and diagnosis of C. pneumoniae infection are often suboptimal in terms of standardization and methodological validation. Respiratory tract samples cultures and polymerase chain reaction (PCR) seem to be the most accurate methods, yet high cost and practical problems render them not widely applicable. In the present case, PCR or cultures of the respiratory tract samples would have not probably contributed to the diagnosis due to the delayed occurrence of the serositis after CAP. The delayed emergence of serositis after the infection could indicate indirect (namely autoimmune) mechanisms. Serologic evaluation is the most frequently used method, either by microimmunofluorescence or ELISA. Standard diagnostic criteria for serologic methods apply, such as the fourfold increase of antibody titers within 4-6 weeks after symptoms onset $[1,22]$. Unfortunately, we did not have baseline values at symptoms onset and during her first hospitalization. However, the patient's C. pneumoniae antibodies kinetics seem to be in line with our assumption of a recent C. pneumoniae CAP. IgM titers presented a peak in our first measurement and then followed a gradual decline, while IgG titers continued to increase within the next 4-6 weeks. (Fig. 1) [1, 22, 23].

Multiple possible mechanisms of cardiac damage have been suggested and could be divided into non-autoimmune and autoimmune mediated pathways [12, 14, 24, 25]. Concerning non-autoimmune pathways, C. pneumoniae-infected macrophages seem to present greater adherence to the endothelium and tend to degenerate easier to foam cells in the presence of LDL cholesterol $[14,23]$. This suggested mechanism implicates that Chlamydia infection could play a role as a cardiovascular disease (CVD) risk factor for atherosclerosis [14, 24]. Autoimmune mechanisms on the other side have been reported to act mainly via molecular mimicry phenomenon [12, 25]. In this case, chlamydial proteins mimic host self-proteins and may trigger systematic autoimmune reactions $[12,25]$. The impressive clinical and biochemistry patient's response to corticosteroids and the lack of any clinical or laboratory response whenever antibiotics were initiated, is indicative of an autoimmune pathogenetic mechanism.

Our study presents several limitations regarding the diagnostic documentation of patient's prior chlamydial infection. Appropriate diagnostic evaluation during the patient's first hospitalization, including PCR/ cultures of respiratory tract samples, as well as baseline antibody titers, was unavailable; however, such a diagnostic work-up is not routinely recommended for uncomplicated CAP. It should be mentioned that the patient presented a rather late serositis a few weeks after her discharge and remission of her initial symptoms. Indeed, similar limitations have been encountered in all relevant published studies (Table 1) [7-11]. These 'inevitable' limitations are somewhat expected in the setting of everyday clinical practice and realworld medicine. Assessment of antibody kinetics in this study might at least partly counteract some of these limitations. In fact, the etiology of polyserositis was determined on the basis of dynamic changes in antibody levels, the clinical course and the response of the patient to steroid and colchicine therapy, and the exclusion of other etiologies. It should be taken into consideration that the aim of this case presentation was to describe a probable rather than definite causative relation of chlamydial infection and pericarditis, and most importantly to raise awareness among doctors regarding the early consideration of this entity in differential diagnosis of similar cases.

In conclusion, C. pneumoniae-associated CVD or autoimmune implications may be more frequent and important than generally thought. Clinicians should be aware of atypical causes in the absence of profound and obvious explanations. Nonsteroidal anti-inflammatory drugs and colchicine constitute the mainstay of therapy in cases of pericarditis. Corticosteroids should be considered in cases of contraindications and/or failure of the above-mentioned regimens or in cases of autoimmune-related serositis.

\section{Abbreviations \\ CAP: Community acquired pneumonia; CVD: Cardiovascular disease; CRP: C-reactive protein; CT: Computed tomography; D: Day; ELISA: Enzyme-linked immunosorbent assay.}

\section{Supplementary Information}

The online version contains supplementary material available at https://doi. org/10.1186/s12890-021-01743-9.

Additional file 1: Graphical presentation of the case report including imaging findings, laboratory findings, and therapeutic strategy.

Acknowledgements

Not applicable.

Authors' contributions

KGK and AK were responsible for treating the patient, conceived the study and drafted the manuscript; GED and EF performed the systematic review and drafted the tables; IPT and NKS participated in the interpretation of data and design of figures; GP participated in the interpretation of data, substantively revised the manuscript, and critically assessed and supervised all stages during the production of this work. All authors read and approved the final manuscript.

Funding

None. 


\section{Availability of data and materials}

The original contributions presented in the study are included in the article/ supplementary material. Further inquiries can be directed to the corresponding author.

\section{Declarations}

\section{Ethics approval and consent to participate}

Ethical review and approval was not required for this study. The patient provided written informed consent to participate in this study.

\section{Consent for publication}

The patient provided written informed consent for study publication.

\section{Competing interests}

The authors declare no competing interests.

Received: 11 July 2021 Accepted: 11 November 2021

Published online: 22 November 2021

\section{References}

1. Sharma L, Losier A, Tolbert T, Dela Cruz CS, Marion CR. Atypical pneumonia: updates on legionella, chlamydophila, and mycoplasma pneumonia. Clin Chest Med. 2017;38(1):45-58. https://doi.org/10.1016/j.ccm.2016.11. 011.

2. Gran JT, Hjetland R, Andreassen AH. Pneumonia, myocarditis and reactive arthritis due to Chlamydia pneumoniae. Scand J Rheumatol. 1993;22(1):43-4. https://doi.org/10.3109/03009749309095111.

3. Fairley CK, Ryan M, Wall PG, Weinberg J. The organisms reported to cause infective myocarditis and pericarditis in England and Wales. J Infect. 1996:32(3):223-5. https://doi.org/10.1016/s0163-4453(96)80023-5.

4. Gnarpe H, Gnarpe J, Gästrin B, Hallander H. Chlamydia pneumoniae and myocarditis. Scand J Infect Dis Suppl. 1997;104:50-2.

5. Odeh M, Oliven A. Chlamydial infections of the heart. Eur J Clin Microbiol Infect Dis. 1992;11(10):885-93. https://doi.org/10.1007/BF01962368.

6. Tong CY, Potter F, Worthington E, Mullins P. Chlamydia pneumoniae myocarditis. Lancet. 1995;346(8976):710-1. https://doi.org/10.1016/ s0140-6736(95)92327-6.

7. Oztek Celebi FZ, Fettah A, Yesil S, et al. Acute haemorrhagic pericarditis: an unusual presentation of Chlamydophila pneumoniae pneumonia infection. Paediatr Int Child Health. 2020;40(3):207-10. https://doi.org/10. 1080/20469047.2020.1728163

8. Suesaowalak M, Cheung MM, Tucker D, Chang AC, Chu J, Arrieta A. Chlamydophila pneumoniae myopericarditis in a child. Pediatr Cardiol. 2009;30(3):336-9. https://doi.org/10.1007/s00246-008-9301-5.

9. Rýzlová M, Gregor P. Acute pericarditis as an organic manifestation of the acute infection Chlamydia pneumoniae. Vnitr Lek. 2008;54(9):866-70.

10. Tenenbaum T, Heusch A, Henrich B, MacKenzie CR, Schmidt KG, Schroten $H$. Acute hemorrhagic pericarditis in a child with pneumonia due to Chlamydophila pneumoniae. J Clin Microbiol. 2005;43(1):520-2. https:// doi.org/10.1128/JCM.43.1.520-522.2005.

11. Zver S, Kozelj M, Cernelc P. Chlamydia pneumoniae pneumonia with acute hemorrhagic pericarditis in patient with acute leukemia. Haematologica. 1997;82(2):254.
12. Bachmaier K, Penninger JM. Chlamydia and antigenic mimicry. Curr Top Microbiol Immunol. 2005;296:153-63. https://doi.org/10. 1007/3-540-30791-5 9.

13. Joshi R, Khandelwal B, Joshi D, Gupta OP. Chlamydophila pneumoniae infection and cardiovascular disease. N Am J Med Sci. 2013;5(3):169-81. https://doi.org/10.4103/1947-2714.109178.

14. Campbell LA, Kuo CC, Grayston JT. Chlamydia pneumoniae and cardiovascular disease. Emerg Infect Dis. 1998;4(4):571-9. https://doi.org/10. 3201/eid0404.980407.

15. Adler Y, Charron P, Imazio M, et al; ESC Scientific Document Group. 2015 ESC Guidelines for the diagnosis and management of pericardial diseases: The Task Force for the Diagnosis and Management of Pericardial Diseases of the European Society of Cardiology (ESC) Endorsed by: The European Association for Cardio-Thoracic Surgery (EACTS). Eur Heart J. 2015;36(42):2921-64. https://doi.org/10.1093/eurheartj/ehv318.

16. Miyashita N, Akaike H, Teranishi H, et al; Atypical Pathogen Study Group. Chlamydophila pneumoniae serology: cross-reaction with Mycoplasma pneumoniae infection. J Infect Chemother. 2013;19(2):256-60. https:// doi.org/10.1007/s10156-012-0494-4.

17. Boonsarngsuk V, Niyompattama A, Teosirimongkol C, Sriwanichrak K. False-positive serum and bronchoalveolar lavage Aspergillus galactomannan assays caused by different antibiotics. Scand J Infect Dis. 2010;42(6-7):461-8. https://doi.org/10.3109/00365541003602064.

18. Danesh J, Collins R, Peto R. Chronic infections and coronary heart disease: is there a link? Lancet. 1997;350(9075):430-6. https://doi.org/10.1016/ S0140-6736(97)03079-1.

19. Mahdi OS, Horne BD, Mullen K, Muhlestein JB, Byrne GI. Serum immunoglobulin $\mathrm{G}$ antibodies to chlamydial heat shock protein 60 but not to human and bacterial homologs are associated with coronary artery disease. Circulation. 2002;106(13):1659-63. https://doi.org/10.1161/01.cir. $0000031567.10814 . d 8$.

20. Vijay A, Stendahl JC, Rosenfeld LE. Mycoplasma pneumoniae pericarditis. Am J Cardiol. 2019;123(8):1383-4. https://doi.org/10.1016/j.amjcard.2019. 01.014.

21. Centers for Disease Control and Prevention. Chlamydia pneumoniae Infection/Treatment. https://www.cdc.gov/pneumonia/atypical/cpneu moniae/hcp/treatment.html (2019). Accessed 25 June 2021.

22. Hvidsten D, Halvorsen DS, Berdal BP, Gutteberg TJ. Chlamydophila pneumoniae diagnostics: importance of methodology in relation to timing of sampling. Clin Microbiol Infect. 2009;15(1):42-9. https://doi.org/10.1111/j. 1469-0691.2008.02075.x.

23. Miyashita N, Kawai Y, Tanaka T, et al. Antibody responses of Chlamydophila pneumoniae pneumonia: why is the diagnosis of $C$. pneumoniae pneumonia difficult? J Infect Chemother. 2015;21(7):497-501. https://doi. org/10.1016/j.jiac.2015.03.003.

24. Stassen FR, Vainas T, Bruggeman CA. Infection and atherosclerosis. An alternative view on an outdated hypothesis. Pharmacol Rep. 2008;60(1):85-92.

25. Díaz F, Collazos J. Myopericarditis due to Chlamydia psittaci. The role of autoimmunity. Scand J Infect Dis. 1997;29(1):93-4. https://doi.org/10. 3109/00365549709008673.

\section{Publisher's Note}

Springer Nature remains neutral with regard to jurisdictional claims in published maps and institutional affiliations. 